\title{
Estudo de Efeitos Ambientais que Influem na Forma da Curva de Lactação de Cabras Mestiças no Estado da Paraíba
}

\author{
Maria Norma Ribeiro ${ }^{1,2}$, Edgard Cavalcanti Pimenta Filho ${ }^{2,3}$
}

\begin{abstract}
RESUMO - O objetivo deste experimento foi estudar a influência de efeitos ambientais sobre a forma da curva de lactação (produção no pico, tempo de pico e persistência) de cabras mestiças Alpina x Gurguéia, ajustada pela função gama incompleta. Dados de 6126 controles, referentes a 877 lactações de 366 cabras criadas na Fazenda Carnaúba, localizada no município de Taperoá, Estado da Paraíba, no período de 1988 a 1995, foram usados. A função foi ajustada por regressão linear, após transformação logarítmica, e pelo método iterativo não-linear. Os parâmetros obtidos por regressão linear tiveram coeficiente de determinação ajustado ( $\left.\mathrm{R}_{\mathrm{a}}^{2}\right)$ de $71 \%$ versus $\mathrm{R}_{\mathrm{a}}^{2}$ de $90 \%$ obtido para a regressão não-linear. Produção máxima, tempo de pico e produção no pico foram obtidos por intermédio da função ajustada por regressão não-linear, que apresentou ajuste superior. O estudo dos efeitos ambientais sobre estas características foi realizado pelo método dos quadrados mínimos, usando um modelo contendo os efeitos de tipo de parto, ordem de parto, ano do parto e estação de parto. Os efeitos ambientes influenciaram todas as características estudadas. Maiores picos de produção foram observados para as cabras adultas, o que foi justificado pelo completo desenvolvimento fisiológico dos animais.
\end{abstract}

Palavras-chave: curva de lactação, cabras, função gama incompleta

\section{Study of the Environmental Effects that Affect the Shape of Lactation Curves of Crossbred Goats in Paraíba State}

\begin{abstract}
The objective of this experiment was to study the effect of environmental factors on the shape of lactation curves (peak yield, time of peak and persistency) of crossbred Alpine x Gurguéia goats fitted by Incomplete gamma function. Data from 6126 records of 877 lactation of 366 goats raised on Carnaúba Farm, Taperoá city, Paraíba State in the period from 1988 to 1995 were used. The function was fitted by linear regression, after logarithmic transformation and by non-linear iterative method. Adjusted coefficient of determination $\left(\mathrm{R}_{\mathrm{a}}^{2}\right.$ ) for linear regression was $71 \%$ versus a $\mathrm{R}_{\mathrm{a}}^{2}$ of $90 \%$ obtained by non-linear regression. Peak yield, time of peak and persistency were obtained by fitting the non-linear regression, that showed superior fit. The study of environmental effects on those traits was made by least squares method, including in the model the following factors: calving type, calving order, calving year and calving season. The environmental effects affected all the studied traits. Higher milk peak yields were observed for adult goats, which was justified by the complete physiological development of these animals.
\end{abstract}

Key Words: lactation curve, goats, incomplete gamma function

\section{Introdução}

Há muito tempo pesquisadores vêm tentando descrever o comportamento da produção de leite ao longo da lactação. Diversos estudos têm sido feitos em bovinos (WOOD, 1967, 1969, 1974), utilizando equações que possibilitem representar a curva de lactação com precisão.

Os modelos matemáticos possibilitam a avaliação de fatores que influem na produção máxima de leite produzida por uma fêmea e dias para se atingir essa produção (MASSELIN et al., 1987; GIPSON e GROSSMAN, 1989; e GENGLER, 1996). As curvas de lactação não somente podem ajudar no manejo do rebanho, principalmente no descarte, na nutrição e na avaliação do estado de saúde dos animais (DUDOUET, 1982), como também são úteis para o desenvolvimento de métodos de comparação entre animais, a partir de lactações incompletas (KEOWN e VAN VLECK, 1973).

Muitos modelos de curva de lactação têm sido propostos e alguns foram testados em cabras (GIPSON e GROSSMAN, 1989; WILLIAMS, 1993a; e RIBEIRO et al., 1997).

O modelo de Wood tem sido usado na maioria dos estudos de curva de lactação, pois permite a estimati-

\footnotetext{
1 Aluno de Doutorado da FCAV/UNESP-Jaboticabal. Bolsista PICD. E-mail: ouricuri@elogica.com.br

2 Prof. do DZ/CCA/UFPB. CAMPUS III-Areia-PB.

${ }^{3}$ Bolsista do CNPq.
} 
va de características básicas da curva, como produção máxima, tempo para se atingir essa produção e persistência, com apenas três parâmetros (WOOD, 1967). É representado por $\mathrm{Y}=\mathrm{b}_{0} \mathrm{x}^{\mathrm{b} 1} \mathrm{e}^{\mathrm{b} 2 \mathrm{x}}$, o qual define uma curva do tipo gama incompleta e pode ser usada em um único animal ou em grupos de animais para representar uma lactação completa. Neste modelo, Y é a produção de leite no tempo $\mathrm{x} ; \mathrm{b}_{0}$, a constante associada a escala de produção do animal; $\mathrm{b}_{1}$, a taxa média de aumento da produção até atingir a produção máxima; $b_{2}$, a taxa média de declínio da produção após a cabra atingir o pico; e e, a base do logaritmo neperiano. Wood propôs o pico de produção ocorrendo em $x=b_{1} / b_{2}$ dias; a produção máxima como $\mathrm{Y}=\mathrm{b}_{0}\left(\mathrm{~b}_{1} / \mathrm{b}_{2}\right)^{\mathrm{b} 1} \mathrm{e}^{-\mathrm{b} 1} \mathrm{~kg}$ de leite; e a persistência, que é a capacidade de um animal manter altos níveis de produção, também definida por WOOD (1967) como a extensão com que o pico é mantido, estimada por $\mathrm{S}=-\left(\mathrm{b}_{1}+1\right) \ln \mathrm{b}_{2}$.

GIPSON e GROSSMAN (1989) relataram que a forma da curva de lactação pode ser influenciada por diversos fatores: ordem de parto, tipo de parto, estação de parto e ano do parto. WOOD (1969) relata que maiores picos são, geralmente, seguidos de decréscimo mais rápido da produção durante a fase descendente, enquanto menores picos são seguidos por decréscimo mais lento da produção ao longo da lactação. WILLIAMS (1993b) relatou que a produção no pico atinge valores máximos entre três e cinco lactações, fato também comprovado por HORAK e PINDAK (1969). A persistência, definida como a taxa com que o pico é mantida, tende a ser maior em cabras mais jovens e diminui com partos sucessivos (GIPSON e GROSSMAN, 1989).

Estudos da influência do tipo de parto sobre a forma da curva de lactação em caprinos são conflitantes. Alguns mostram influência significativa deste efeito sobre a forma da curva de lactação de cabras (MAVROGENIS et al., 1984). Entretanto, WILLIAMS (1993b) e WAHOME et al. (1994) não encontraram efeito significativo do tipo de parto sobre a forma da curva de lactação de cabras e comentam que em sistema de produção comercial esse efeito é de pouca importância prática.

Os objetivos deste estudo foram avaliar a influência do tipo de parto, da estação de parto, ano e da ordem do parto sobre a forma da curva de lactação de cabras, usando a função Gama Incompleta, e comparar a qualidade do ajuste proporcionado pela função na sua forma normal e linearizada por intermédio do coeficiente de determinação ajustado.

\section{Material e Métodos}

Foram utilizados no presente estudo dados referentes a 6126 controles de produção de leite de 877 lactações de 366 cabras mestiças Alpina x Gurguéia, de composição genética variada, controladas no período de 1988 a 1995, de propriedade da Fazenda Carnaúba, município de Taperoá, Estado da Paraíba. Os animais da raça Gurguéia são homólogas das Alpinas, porém apresentam baixa capacidade leiteira, devido ao processo de seleção natural por que passaram ao longo de sucessivas gerações para se adaptarem à região semi-árida.

A Fazenda está localizada na microrregião do Cariri Ocidental, a $7^{0} 12^{\prime} 23^{\prime \prime}$ de latitude sul e $36^{0} 49^{\prime} 25^{\prime}$ de longitude W.G., à altitude de $500 \mathrm{~m}$ (IBGE, 1990).

O clima da região está incluído no subtipo B, subdesértico quente, de tendência tropical, com precipitação em torno de $300 \mathrm{~mm}$ anuais. Apresenta curta estação chuvosa e estação seca prolongada, maior que 8 meses, e umidade relativa do ar em torno de $65 \%$ (Governo do Estado da Paraíba/UFPB, 1985).

Os animais foram criados em sistema semi-extensivo e alimentam-se de Capim Buffel (Cenchrus ciliaris $L$.) e pasto nativo na época chuvosa e capim elefante (Pennisetum purpureum schum.), palma forrageira (Opuntia sp.), raspa de mandioca (Manihot sativa), bagaço de cana hidrolisado (Sacharum oficcinarum) e concentrado protéico na época seca. Utiliza-se o sistema de monta natural controlada e controle sanitário, sistematicamente. O controle leiteiro foi feito a cada 28 dias, em duas ordenhas diárias, com intervalo de 12 horas entre ordenhas. A produção de leite foi anotada em fichas individuais, nas quais também são feitas anotações referentes à vida reprodutiva de cada animal. A partir das fichas de produção e reprodução, foi editado um arquivo contendo número da cabra, data do parto, produção de leite, tipo de parto, ordem e estação de parto. Foram eliminadas as lactações que iniciaram o controle de produção 35 dias após o parto e lactações com menos de cinco controles por lactação, ficando-se com 877 lactações para ajuste da curva de lactação, das quais apenas 657 estimaram curvas típicas e foram utilizadas na análise de variância dos efeitos ambientais.

Os dados foram classificados em estádios com intervalos de 28 dias (freqüência com que os controles foram realizados).

Os parâmetros da Função Gama Incompleta na sua forma normal $\left(\mathrm{y}=\mathrm{b}_{0} \mathrm{x}^{\mathrm{b} 1} \mathrm{e}^{-\mathrm{b} 2 \mathrm{X}}\right)$ foram obtidos por técnicas não-lineares pelo método de Gauss-Newton, disponível no PROC NLIN do SAS (1992), e pelo 
método dos quadrados mínimos ordinários em análise de regressão utilizando-se o PROC REG do SAS (1992) a partir da função linearizada Lny $=\operatorname{lna}+$ blnx $-c x$, em que y e produção de leite $(\mathrm{kg})$; $\mathrm{x}$, estádio da lactação; e a, b e c são parâmetros da curva a serem estimados. O tempo de pico, a produção no pico e a persistência foram obtidos como sugeridos por WOOD (1967).

O melhor método de ajuste foi escolhido pelo valor do Coeficiente de Determinação $\operatorname{Ajustado}\left(\mathrm{R}_{\mathrm{a}}{ }^{2}\right)$.

$$
R_{a}^{2}=\frac{(n-1) R^{2}-p}{n-p-1}
$$

em que

$P=$ número de parâmetros do modelo;

$n$ =número de observações; e

$R^{2}=$ coeficiente de determinação.

$\mathrm{O}$ estudo dos fatores de meio que influenciam a forma da curva de lactação por meio das características produção máxima na lactação, época em que o pico ocorreu e persistência da lactação, foi feito pelo método dos quadrados mínimos, por intermédio do PROC GLM do SAS (1992), a partir do seguinte modelo matemático:

$$
\begin{aligned}
& \mathrm{Y}_{\mathrm{ijklm}}=\mu+\mathrm{TP}_{\mathrm{i}}+\mathrm{EP}_{\mathrm{j}}+\mathrm{AP}_{\mathrm{k}}+\mathrm{OP}_{1}+\varepsilon_{\mathrm{ijkl} \mathrm{m}} \\
& \text { em que } \\
& \mathrm{Y}_{\mathrm{ijklm}}=\text { é a observação (produção máxima, per- }
\end{aligned}
$$
sistência e tempo para se atingir a produção máxima), referente à lactação $\mathrm{m}$, ocorrido no parto 1 , no ano $\mathrm{k}$, na estação $\mathrm{j}$, do tipo de parto i;

$\mu=$ constante comum a todas as observações;

$\mathrm{TP}_{\mathrm{i}}=$ efeito fixo do tipo de parto $(\mathrm{i}=1$-simples e 2múltiplo);

$\mathrm{EP}_{\mathrm{j}}=$ efeito fixo da estação de parto $(\mathrm{j}=1$ - águas, 2-seca e 3-meia estação);

$\mathrm{AP}_{\mathrm{k}}=$ efeito fixo do ano de parto $(\mathrm{k}=1988,1989$, 1990, 1991, 1992, 1993, 1994, 1995);

$\mathrm{OP}_{1}=$ efeito fixo da ordem de parto (1=1-primeiro, 2-segundo, 3-terceiro, 4-quarto, 5-quinto, 6-seis ou mais); $\mathrm{e}$

$\varepsilon_{\mathrm{ijkl} \mathrm{m}}=$ erro associado a cada observação pressupondo-se NID $\left(0, \sigma^{2}\right)$.

As estações de parto foram determinadas de acordo a distribuição das chuvas. Assim, os meses de outubro a janeiro correspondem à estação seca; os meses de fevereiro a abril, à época das chuvas; e os meses de junho a agosto, à época intermediária, com boa disponibilidade de alimentos.

\section{Resultados e Discussão}

O Coeficiente de Determinação Ajustado $\left(\mathrm{R}_{\mathrm{a}}{ }^{2}\right)$ obtido para a Função Gama Incompleta na sua forma normal foi de $90 \%$ e, na sua forma linearizada, $71 \%$.

Estas diferenças podem estar associadas aos vícios gerados pela transformação logarítmica. Segundo KELLOG et al. (1977) e CONGLETON e EVERETT (1980), a transformação logarítmica leva em conta a suposição de maior variabilidade em torno do pico que nos extremos da curva, o que gera diferentes variâncias que podem contribuir para a obtenção de estimativas viesadas. GIRON PINEDA (1987) observou que a linearização pode gerar assíntotas verticais, causando aumento ou diminuição na estimativa da produção de leite de forma infinita. Esse autor afirma que problemas dessa natureza são ainda maiores quando o número de pontos disponíveis para o ajuste é pequeno; é o caso, por exemplo, das situações em que se trabalha com dados de controle mensal. As técnicas não-lineares, por outro lado, consideram igual variação durante toda lactação de modo que as curvas estimadas usando este método apresentam menores vícios.

LOPES (1996) fez ajuste da função Gama Incompleta por regressão linear e não linear e obteve $\mathrm{R}_{\mathrm{a}}{ }^{2}$ bastante superior neste último caso, indicando que o ajuste de funções não lineares, após linearização dos parâmetros, não é a forma mais adequada, já que a qualidade do ajuste diminui sensivelmente. Com base nessas informações, o estudo dos efeitos ambientais sobre a forma da curva de lactação do rebanho foi feito a partir dos parâmetros obtidos por processo iterativo, em regressão não linear.

Na Tabela 1 encontram-se os resultados da análise de variância das características produção máxima, tempo para atingir o pico e persistência da curva de lactação. O coeficiente de determinação ajustado $\left(\mathrm{R}_{\mathrm{a}}{ }^{2}\right)$ obtido para a curva média foi de $90 \%$. Foram obtidas 248 curvas atípicas, cujos valores $b_{0}, b_{1}$ ou $b_{2}$ foram negativos. O grande número de curvas atípicas obtidas no presente estudo deve estar relacionado ao tipo de controle adotado no rebanho que é mensal, de modo que a cabra pode ter passado do pico de produção, quando da realização do primeiro controle. A curva média do rebanho não apresentou pico, o que também pode ter contribuído para o alto número de curvas atípicas obtidas. Estudos têm mostrado que a porcentagem de curvas atípicas obtidas tende a ser baixa em sistemas de controle leiteiro mais freqüente, principalmente quando o primeiro controle é feito antes de 10 dias após o parto (CONGLETON e EVERETT, 1980).

A Tabela 2 apresenta as médias de quadrados mínimos das características da curva de lactação. A média de produção máxima variou de 1,33 a 2,30 kg 
RIBEIRO e PIMENTA FILHO

Tabela 1- Quadrados médios das características de produção máxima (PM), tempo de pico (TP) e persistência da lactação (P) de cabras mestiças

Table 1 - Mean squares of the traits of maximum production (MP), time of peak (TP) and persistency of the lactation $(P)$ of crossbred goats

\begin{tabular}{|c|c|c|c|c|}
\hline \multirow[t]{2}{*}{$\begin{array}{l}\text { Fonte de variação } \\
\text { Source of variation }\end{array}$} & \multirow[t]{2}{*}{$\begin{array}{l}\text { gl } \\
d f\end{array}$} & \multicolumn{3}{|c|}{$\begin{array}{l}\text { Quadrados médios } \\
\text { Mean squares }\end{array}$} \\
\hline & & $\begin{array}{l}\text { PM }(\mathrm{kg}) \\
\text { MP }\end{array}$ & $\begin{array}{l}\text { TPI (dias) } \\
\text { TP (days) }\end{array}$ & $\mathrm{S}$ \\
\hline $\begin{array}{l}\text { Tipo de parto } \\
\text { Birth type }\end{array}$ & 1 & $10,17652 * *$ & $2024,8321^{* *}$ & $7,54426^{* *}$ \\
\hline $\begin{array}{l}\text { Ordem do parto } \\
\text { Calving order }\end{array}$ & 5 & $45,55406^{* *}$ & $4161,7951^{* *}$ & $3,46879 * *$ \\
\hline $\begin{array}{l}\text { Ano de parto } \\
\text { Calvingyear }\end{array}$ & 7 & $51,08551 * *$ & $6275,4743 * *$ & $46,12609^{* *}$ \\
\hline Estação de parto & 2 & $27,51213^{* *}$ & $14136,5734^{* *}$ & $34,82397 * *$ \\
\hline $\begin{array}{l}\text { Calving season } \\
\mathrm{R}^{2} \\
\mathrm{CV}(\%)\end{array}$ & & $\begin{array}{r}0,45 \\
26\end{array}$ & $\begin{array}{r}0,13 \\
56\end{array}$ & $\begin{array}{r}0,15 \\
14\end{array}$ \\
\hline
\end{tabular}

$* *=\mathrm{P}<0,01$.

Tabela 2 - Médias estimadas das características, produção máxima de leite (PM), tempo de pico (TP) e persistência da lactação, para cada classe

Table 2 - Estimated mean of the traits, maximum production (MP), time of peak (TP) and persistency of lactation (P), for each class

\begin{tabular}{|c|c|c|c|c|}
\hline Efeito & $\mathrm{N}$ & PM $(\mathrm{kg})$ & TP (dias) & $\mathrm{P}$ \\
\hline \multirow[t]{3}{*}{ Effect } & & $M P$ & & \\
\hline & & Média \pm D.P. & Média \pm D.P. & Média \pm D.P. \\
\hline & & Mean \pm S.D. & Mean \pm S.D. & Mean \pm S.D. \\
\hline \multicolumn{5}{|l|}{ Tipo de parto } \\
\hline \multicolumn{5}{|l|}{ Birth type } \\
\hline Simples & 2134 & $1,87 \pm 0,02^{\mathrm{a}}$ & $35,48 \pm 0,64^{\mathrm{a}}$ & $6,39 \pm 0,03^{a}$ \\
\hline \multicolumn{5}{|l|}{ Single } \\
\hline Múltiplo & 2060 & $2,12 \pm 0,02^{b}$ & $32,36 \pm 0,66^{\mathrm{b}}$ & $6,28 \pm 0,03^{b}$ \\
\hline \multicolumn{5}{|l|}{ Multiple } \\
\hline \multicolumn{5}{|l|}{ Ordem de parto } \\
\hline \multicolumn{5}{|l|}{ Calving order } \\
\hline 1 & 686 & $1,33 \pm 0,03^{\mathrm{e}}$ & $41,49 \pm 1,13^{\mathrm{a}}$ & $6,56 \pm 0,05^{a}$ \\
\hline 2 & 769 & $1,84 \pm 0,03^{\mathrm{d}}$ & $34,05 \pm 1,02^{c}$ & $6,29 \pm 0,05^{a}$ \\
\hline 3 & 517 & $2,09 \pm 0,04^{\mathrm{c}}$ & $28,06 \pm 1,23^{\mathrm{e}}$ & $6,11 \pm 0,06^{\mathrm{c}}$ \\
\hline 4 & 588 & $2,30 \pm 0,03^{\mathrm{a}}$ & $30,64 \pm 1,16^{\mathrm{d}}$ & $6,17 \pm 0,05^{c}$ \\
\hline 5 & 474 & $2,13 \pm 0,04^{\mathrm{c}}$ & $37,76 \pm 1,30^{\mathrm{b}}$ & $6,53 \pm 0,06^{a}$ \\
\hline$>6$ & 1160 & $2,22 \pm 0,03^{b}$ & $32,17 \pm 0,84^{\mathrm{c}}$ & $6,34 \pm 0,04^{b}$ \\
\hline \multicolumn{5}{|c|}{ Estação de parto } \\
\hline \multicolumn{5}{|c|}{ Calving season } \\
\hline Seca & 1466 & $1,75 \pm 0,02^{\mathrm{a}}$ & $35,61 \pm 0,76^{\mathrm{a}}$ & $6,31 \pm 0,03^{a}$ \\
\hline \multicolumn{5}{|l|}{ Dry } \\
\hline Águas & 1290 & $1,98 \pm 0,02^{b}$ & $29,36 \pm 0,78^{b}$ & $6,11 \pm 0,03^{b}$ \\
\hline \multicolumn{5}{|l|}{ Rainy } \\
\hline Meia estação & 1438 & $2,24 \pm 0,02^{\mathrm{c}}$ & $36,36 \pm 0,76^{\mathrm{c}}$ & $6,35 \pm 0,03^{c}$ \\
\hline \multicolumn{5}{|l|}{ Middle season } \\
\hline \multicolumn{5}{|l|}{ Ano de parto } \\
\hline \multicolumn{5}{|l|}{ Calvingyear } \\
\hline 1988 & 629 & $2,59^{\mathrm{a}}$ & $36,88^{\mathrm{a}}$ & $6,59^{\mathrm{a}}$ \\
\hline 1989 & 909 & $1,92^{\mathrm{c}}$ & $38,15^{\mathrm{a}}$ & $6,45^{c}$ \\
\hline 1990 & 914 & $2,27^{\mathrm{b}}$ & $24,63^{\mathrm{c}}$ & $5,84^{e}$ \\
\hline 1991 & 448 & $1,67^{d}$ & $37,71^{\mathrm{a}}$ & $6,66^{\mathrm{a}}$ \\
\hline 1992 & 444 & $1,72^{\mathrm{d}}$ & $39,49^{a}$ & $6,56^{\mathrm{a}}$ \\
\hline 1993 & 643 & $1,65^{\mathrm{d}}$ & $34,34^{b}$ & $6,32^{\mathrm{cd}}$ \\
\hline 1994 & 64 & $1,29^{\mathrm{e}}$ & $26,32^{c}$ & $5,88^{\mathrm{e}}$ \\
\hline 1995 & 143 & $1,67^{\mathrm{d}}$ & $26,55^{\mathrm{c}}$ & $6,19^{d}$ \\
\hline
\end{tabular}

Médias, dentro de cada efeito, seguidas de letras diferentes são diferentes $(P<0,05)$ pelo teste Tukey.

Means, within each effects, followed by different letters, are different $(P<.05)$ by Tukey test. 
872 Rev. bras. zootec.

de leite, com pico ocorrendo entre 24 e 42 dias após o parto. A persistência, por sua vez, variou de 5,84 a 6,66 . Estes valores são expressos sem unidades de medida e maiores valores absolutos estão relacionados com lactações mais persistentes (EL FARO, 1996). Esses apresentam limitação quanto a sua interpretação prática, o que dificulta qualquer avaliação. A razão entre produções acumuladas em duas fases da lactação, expressa em porcentagem, tem sido alternativa utilizada pelos pesquisadores (MONTALDO et al., 1997) para resolver esse inconveniente. Esta forma de cálculo é mais real e permite avaliar a intensidade com que a cabra se mantém produzindo ao longo de toda a lactação. A baixa média de duração da lactação do rebanho estudado impossibilitou o uso desse procedimento.

Altos coeficientes de variação foram observados para a produção máxima e tempo para atingir o pico. Entretanto, a persistência apresentou coeficiente de variação muito baixo como mostra a Tabela 1. Nesta Tabela observa-se que o tipo de parto influenciou significativamente as características tempo de pico, produção máxima e persistência da lactação $(\mathrm{P}<0,01)$.

As cabras que tiveram partos múltiplos apresentaram maiores picos de produção em relação as que tiveram partos simples, as quais apresentaram lactações mais persistentes e picos ocorrendo mais cedo (Tabela 2). Entretanto, a maioria dos estudos considera este efeito de pouca importância para a forma da curva de lactação (WILLIAMS, 1993b; WAHOME et al., 1994). As curvas para os diferentes tipos de parto são representadas na Figura 1, em que se observa diferença apenas na escala, mas não na forma das curvas estimadas para cada tipo de parto.

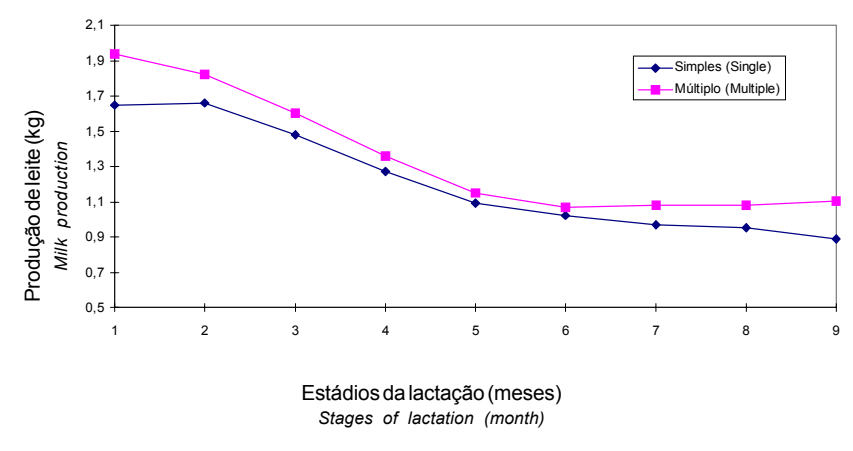

Figura 1 - Curva de lactação estimada por meio da função gama incompleta, de acordo com o tipo de parto.

Figure 1 - Estimated lactation curve by incomplete gamma function according to the birth type.
Observou-se efeito significativo da ordem de parto sobre a produção máxima, tempo de pico e persistência da lactação $(\mathrm{P}<0,01)$. As cabras de primeiro parto apresentaram menor produção no pico, fato também observado por HORAK e PINDAK (1969). Pela Figura 2 observa-se que as cabras do quarto ao sexto partos apresentaram curva de lactação semelhante, porém as cabras de terceiro e quinto partos apresentaram produção no pico semelhantes (Tabela 2). Estes resultados concordam com WILLIAMS (1993b), que observou maiores picos de produção para cabras acima do terceiro parto, o que se deve ao fato de que, nesta fase, as cabras já atingiram todo o seu desenvolvimento fisiológico.

$\mathrm{O}$ ano de parto influenciou significativamente todas as características da curva de lactação estudadas $(\mathrm{P}<0,01)$, como mostra a Figura 2. Observa-se que a produção no pico apresentou tendência de diminuição ao longo dos anos, o que se deve ao fato de que, neste período, foram incorporados animais mais jovens no rebanho, além do longo período de estiagem ocorrido nesta época, que contribuiu para a diminuição na produtividade do rebanho. Graves problemas sanitários também ocorreram neste período, principalmente no ano de 1994, dizimando quase todo o rebanho. As cabras paridas no ano de 1990 atingiram o pico de produção mais cedo com a menor persistência; as paridas em 1988 tiveram lactações mais persistentes, maior produção no pico e pico ocorrendo mais cedo (Tabela 2). Estudos com bovinos mostraram influência significativa do ano de parto sobre a forma da curva de lactação, que foi atribuída a fatores climáticos e de manejo sanitário e alimentar (QUEIROZ et al., 1991). A estação de parto influenciou significativamente todas as características da curva de lactação $(\mathrm{P}<0,01)$. Maiores produção no pico, tempo de pico e persistência da lactação foram obtidos para cabras paridas na meia estação, época correspondente ao final das chuvas, quando o pasto é abundante. É esperado que animais mais persistentes tenham menores picos de produção, como observado por MONTALDO et al. (1997) e GIPSON e GROSSMAN (1990). Por outro lado, dentro da mesma estação, foi observada maior média de persistência para cabras que tiveram maior pico de produção. Na estação chuvosa, ocorre inibição do apetite e os animais tendem a se alimentar menos. Já na meia estação, além do pasto ser abundante, o clima favorece a procura de alimentos pelos animais, o que estimula a produção de leite, resultando em maiores níveis produtivos, como mostra a Figura 3. Entretan- 


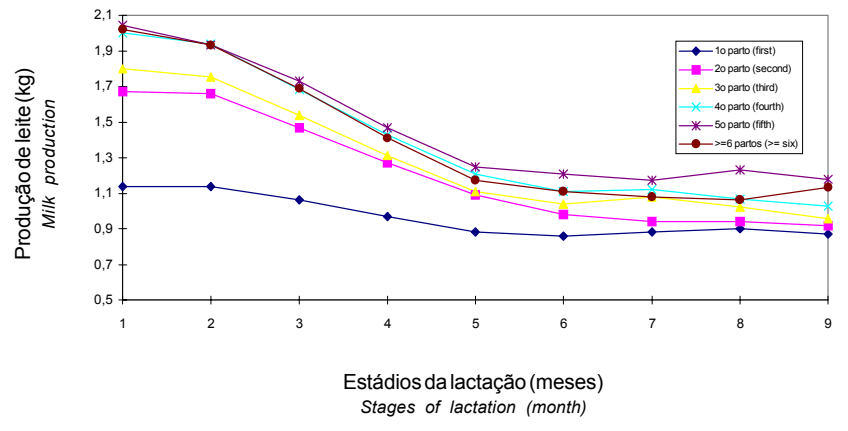

Figura 2 - Curva de lactação estimada por intermédio da função gama incompleta de acordo com a ordem de parto.

Figure 2 - Estimated lactation curve by incomplete gamma function according to the calving order.

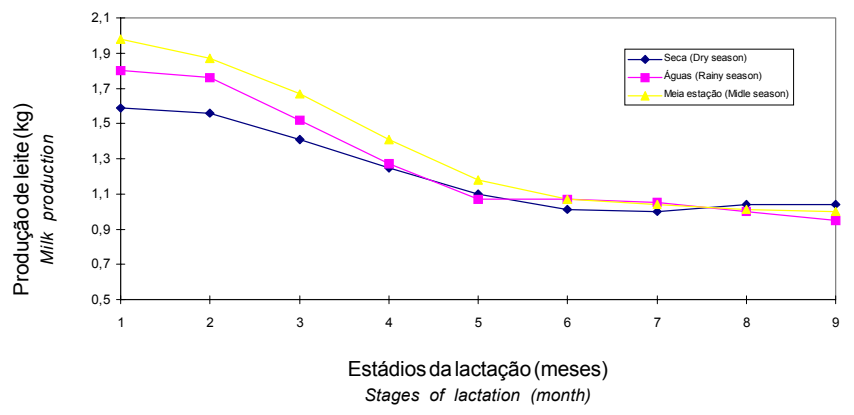

Figura 3 - Curva de lactação estimada por intermédio da função gama incompleta, de acordo com a estação de parto.

Figure 3 - Estimated lactation curve by incomplete gamma function according to the calving season.

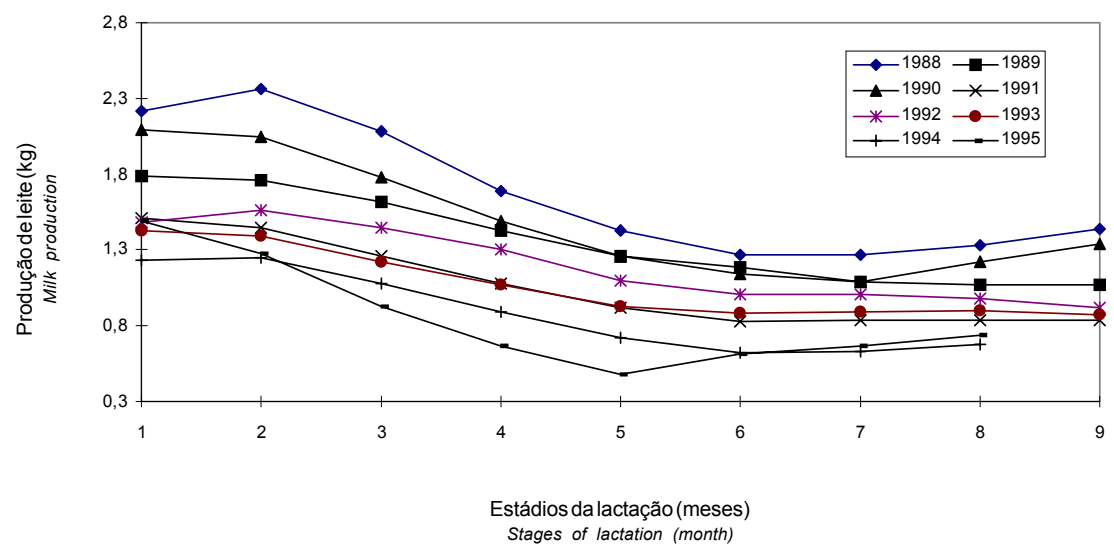

Figura 4 - Curva de lactação estimada por intermédio da função gama incompleta, de acordo com o ano do parto.

Figure 4 - Estimated lactation curve by incomplete gamma function according to the calving year.

to, a diferença em produção entre as três estações ocorreu apenas até o sexto estádio da lactação (cerca de 150 dias). Após essa fase, a produção de leite se manteve no mesmo nível nas três estações.

Em cabras estabuladas, WILLIAMS (1993b) não observou efeito significativo da estação de parto sobre a persistência da lactação, em razão de a disponibilidade de alimentos ser a mesma nas diferentes estações.

\section{Conclusões}

Todos os efeitos ambientais estudados influenciaram a forma da curva de lactação.

$\mathrm{O}$ uso da função gama incompleta para identifi- car falhas no manejo de um rebanho pode ser viável, desde que usada para subgrupos de lactações em animais de mesma ordem de parto, paridos no mesmo ano e estação.

Os valores de persistência obtidos pela função gama incompleta são bastante limitados, pois não permitem avaliar a sua intensidade.

Em rebanhos com lactações mais longas, a persistência poderia ser obtida como proporção entre produções acumuladas em duas fases da lactação.

Os vícios gerados nas estimativas dos parâmetros obtidos a partir de modelos linearizados podem ser minimizados com o uso de métodos nãolineares de estimação. 


\section{Referências Bibliográficas}

DUDOUET, E., 1982. Courbe de lactation théorique de la chèvre et applications. Le Point Vétérinaire, 14:53-61.

CONGLETON JR., W. R., EVERETT, R. W., 1980. Error and bias in using the incomplete gamma function. J. Dairy Sci., 63:101-108.

DRAPER, N.M., SMITH, H. 1981. Applied regression analysis. 2.ed., New York: Wiley.

EL FARO, L. 1996. Estudo da curva de lactação de um rebanho da raça caracu. Jaboticabal: UNESP/FCAVJ. 179p.

GENGLER, N. 1996. Persistency of lactation yields: a review. Proceedings of the Interbull Annual Meeting. Genbloux, Belgium, 21-23 January, Bulletin n.12, Dept ${ }^{\circ}$ of Animal Breeding and Genetics, SLU., Uppsala, Sweden. p.87-96.

GIPSON, T.A., GROSSMAN, M., 1989. Diphasic analysis of lactation curves in dairy goats. J. Dairy Sci., 72:1035-1044.

GIRON PINEDA, A.M. Mathematical functions applied to dairy cow lactation curves. University of Flórida, Flórida. 1987. 243p. (Master of Science).

GOVERNO ESTADO DA PARAÍBA. SEC. Educação. UFPB. Atlas Geográfico do Estado da Paraiba, Grafset. 1985. 100p.

HORAK, F., PINDAK, A. 1969. A study on the lactation persistence with goats. Acta. Univ. Agric. Fac. Agron., 17:299-305.

IBGE-Enciclopédia dos Municípios Brasileiros. Inst. de Geografia e Estatística, v. XVII, 1990.

KEOWN, J.F., VAN VLECK, L.D. 1973. Extending lactation records in progress to 305 day equivalent. J. Dairy Sci., 56:1070-1079.

KELLOG, D.W., URQUHART, N.S., ORTEGA, A.J. 1977. Estimating Holstein lactation curves with a gama curve. $J$. Dairy Sci., 60:1308-1315.

LOPES, M.A., VEIGA, R.D., NEIVA, R.S. et al. Aplicação dos métodos dos quadrados mínimos e regressão não linear para ajustar curvas de lactação de vacas holandesas. In: REUNIÃO ANUAL DA SOCIEDADE BRASILEIRA DE ZOOTECNIA, 45. Fortaleza, 1996. Anais...Fortaleza: SBZ, 1996. p.66-67.

MASSELIN, S., SAVANT, D., CHAPPOUTOR et al. 1987. Adjustment models for lactation curves. Ann. Zootech., 36:171-206.

MAVRogenis, A.P., CONSTATINOU, A., LOUCA, A., 1984. Environmental and genetic causes of variation in production traits of Damascus goats. Anim. Prod., 38:91-97.

MONTALDO, H., ALMANZA, A., JÚAREZ, A. 1997. Genetic group, age and season effects on lactation curve shape in goats. Small Rum. Res., 24(3):195-202.

QUEIROZ, S.A., FREITAS, M.A.R. de, ALBUQUERQUE, L.G. et al. 1991. Fatores genéticos e de ambiente que influenciam os componentes da curva de lactação de bovinos da raça holandesa. Arq. Bras. Med. Vet. Zootec., 43(4):357-370.

RIBEIRO, M.N., ALBUQUERQUE, L.G., PIMENTA FILHO, E.C. Comparação de funções matemáticas no ajuste da curva de lactação de cabras mestiças no Cariri Paraibano. In: REUNIÃO ANUAL DA SOCIEDADE BRASILEIRA DE ZOOTECNIA, 34. Juiz de Fora, MG. Anais...Viçosa: SBZ p.272-274, 1997.

SAS. USER'S GUIDE: Statistics. SAS Institute. Inc., Cary, North Caroline. 1992.

WAHOME, R. G.,CARLES, A. B., SCHWARTZ, H. J. 1994. An analysis of the variation of the lactation curve of small East African Goats. Small Rum. Res., 15:1-7.

WILLIAMS, J.C. 1993a. An empirical model for the lactation curve of white British dairy goats. Anim. Prod., 57:91-97.

WILLIAMS, J.C. 1993b. Influence of farm, parity, season and litter size on the lactation curve parameters of white British dairy goats. Anim. Prod., 57:99-104.

WOOD, P.D.P. 1967. Algebraic model of the lactation curve in Cattle. Nature, 206:164-165.

WOOD, P.D.P. 1969. Factors affecting the shape of the lactation curve. Anim. Prod.,11:307-316.

WOOD, P.D.P. 1974. A note on the estimation of total lactation yield from production on a single day. Anim. Prod., 16:393-396.

Recebido em: 09/06/97

Aceito em: 26/01/99 